\title{
Active Safety Planning Method for Transportation Network Design
}

\author{
Hongzhi Lin $(10)$ \\ School of Economics and Management, Southeast University, Nanjing 211189, China \\ Correspondence should be addressed to Hongzhi Lin; linhz@seu.edu.cn
}

Received 6 January 2021; Revised 20 June 2021; Accepted 14 November 2021; Published 26 November 2021

Academic Editor: Zhile Yang

Copyright $\odot 2021$ Hongzhi Lin. This is an open access article distributed under the Creative Commons Attribution License, which permits unrestricted use, distribution, and reproduction in any medium, provided the original work is properly cited.

\begin{abstract}
Traffic accidents are frequent although various countermeasures are introduced. Traffic safety cannot be fundamentally improved if it is not considered in the transportation network design stage. Although it is well known that traffic safety is one of the most important concerns of the public, traffic safety is not adequately accommodated in transportation planning. This paper considers traffic safety as a major criterion in designing a transportation network. It is a kind of proactive measure rather than reactive measure. A bilevel programming model system is proposed where the upper level is the urban planners' decision to minimize the estimated total number of traffic accidents, and the lower level is the travelers' response behaviors to achieve transportation system equilibrium. A genetic algorithm (GA) with elite strategy is proposed to solve the bilevel model. The method of successive averages (MSA) is embedded for the lower level model, which is a feedback procedure between destination choice and traffic assignment. To demonstrate the effectiveness of the proposed method and algorithm, an experimental study is carried out. The results show that these methods can be a valuable tool to design a safer transportation network although efficiency, in terms of system total travel time, is slightly sacrificed.
\end{abstract}

\section{Introduction}

The increasing concern of traffic safety issues has resulted in integrating the safety factors in the transportation network design models instead of only focusing on congestions and environments. In 1998, the Transportation Equity Act for the 21st Century of United States clearly required that the urban planning departments should consider road traffic safety an important factor in the whole process of transportation planning (TEA-21, 1998). The conventional traffic safety countermeasures are to diagnose and identify dangerous link sections and black spots through the statistical analysis of occurring accidents. It is a kind of reactive countermeasures after building roads and serious accidents and is usually noted as passive traffic safety. Because the reactive measures are mostly limited to the means of traffic engineering, they are not effective in case of many traffic accidents resulting from transportation planning. Therefore, the problem of frequent traffic accidents cannot be fundamentally solved. With the study of passive traffic safety, researchers began to realize that traffic safety should be accommodated in advance, especially at the transportation network design stage [1]. It could be an effective approach to improve the safety level of road network. This preventive measure is noted as active safety planning. It is worthwhile to focus more on designing a safe road network at the very beginning. In fact, the Safe, Accountable, Flexible, Efficient Transportation Equity Act of United Stated requires that various transportation planning departments take the safety level into consideration in every step of transportation planning [2].

However, few previous researches have been conducted to investigate active safety planning at the transportation network design stage. Yang et al. [3] proposed a three-objective bilevel programming model for optimizing variable speed limits to achieve better road network performance in terms of environment, efficiency, and safety. It is a kind of transportation operations for built road network, where the decision variables are variable speed limits. The link traffic safety is measured by the number of expected road accidents that is a power function of link traffic volume. The parameters are assumed to be the same for all links for 
simplification. The simplified safety performance function is used to demonstrate the proposed method, and it is suggested to be replaced by another realistic form. The lower level model is a deterministic user equilibrium model for traffic assignment, while trip distribution is not considered. $\mathrm{Xu}$ et al. [4] proposed a multiobjective bilevel programming model for stochastic network design problem with fuzzy goals. Four objectives were considered, including environment, efficiency, traffic safety, and construction cost. It is a kind of continuous network design where the decision variables are link capacity enhancements. The total number of traffic accidents is equal to the total vehicle miles traveled (VMT) multiplied by a fixed accident rate, which is not realistic. A conventional traffic assignment is adopted in the lower-level model, while the trip distribution is not considered. Rashidi et al. [5] proposed a mixed-integer bilevel programming model for locating sidewalks and crosswalks to minimize network cost where a lack of safety is penalized with a dollar-value equivalent. In the upper level, the urban planners decide on the location of traffic calming facilities, and in the lower level, the travelers decide on the travel mode and route. The lower level problem is a conventional traffic assignment problem with user equilibrium. Haas and Bekhor [6] proposed a biobjective bilevel programming model for the network design problem to achieve travel time minimization and road safety maximization. In the upper level, the link safety performance is measured by a negative binomial function. It is a zero-one integer programming where the decision variables are lanes addition or not. In the lower level, it is a conventional traffic assignment represented by deterministic user equilibrium. Zhong et al. [7] examined how road pricing affects both traffic efficiency and automobile safety under demand uncertainty. A biobjective bilevel programming model was developed for the traffic accident minimization pricing problem. They demonstrated that the efficiency-oriented road pricing is less beneficial to accident reduction. The traffic accident rates were formulated as a quadratic function of volume-to-capacity. It is a kind of passive safety measures with built road network as the decision variables are road pricing. The lower level is also a conventional traffic assignment where destination choice is not considered. Possel et al. [8] proposed a multiobjective bilevel programming model for network design problem. Three externalities are minimized, which are total travel time, total number of traffic accidents, and total exhaust emission. A fixed accident rate is used to generate the total number of traffic accident fatalities, which is rough. The lower level is operationalized by solving a conventional static user equilibrium problem. The results show that the genetic algorithm outperforms the simulated annealing algorithm. Lin and Wei [9] noticed that traffic safety is usually overlooked at network design stage. They proposed a bilevel programming model for network design problem to achieve both traffic safety and risk equity. It is a kind of continuous network design that is not applicable in practice.

In summary, the contribution of this paper is four folds. First, the safety performance is accommodated in the transportation network stage. It is a kind of proactive measure rather than reactive measure. It can reduce traffic accidents from the very beginning. Second, a nonlinear integer programming is formulated for mixed transportation network design in the upper level. It is the decision of expanded location and lane numbers. Third, there is a transportation system equilibrium to represent travel response behaviors in the lower level. It is a feedback procedure between destination choice and traffic assignment, rather than traffic assignment only. Lastly, a genetic algorithm with elite strategy is proposed for the bilevel programming model system. Method of successive averages (MSA) is embedded for the lower level model to reach transportation system equilibrium.

The remainder of this article is organized as follows. The proposed methodology is formulated in Section 2. It is a bilevel model system where the upper level is to minimize the total number of traffic accidents given investment budget, and the lower level is traveler response behaviors to the upper level decisions. The detailed solution algorithm is described in Section 3. It is a genetic algorithm with elite strategy where MSA is embedded for the lower level model. In order to demonstrate the effectiveness of the model and algorithm, an experimental study is carried out in Section 4. Section 5 concludes this paper.

\section{Methodology}

Transportation network design problem is well known to be a Stackelberg game with leader-follower decision structure. It is conventionally formulated as a bilevel model framework. The urban planners design road network to optimize a certain network performance in the upper level, and the travelers make travel decisions to maximize individual utilities in the lower level. The lower level decisions are made after the upper level decisions. However, the upper level must take the behavior responses of the lower level into account to adjust decisions. The feedback process repeats until expected network performances are optimized.

This paper aims to propose a method and an algorithm for safe transportation network design. The bilevel model framework is shown in Figure 1. It can explicitly capture the leader-follower nature of the relationship between urban planners and travelers. The upper level is to optimize network safety performance with investment constrains. The lower level is a feedback procedure between trip distribution and traffic assignment. It is usually termed as transportation system equilibrium. The detailed models are elaborated in the following sections.

2.1. The Upper Level Model. There are few methods to estimate network safety performance in transportation planning. Usually, a fixed accident rate is adopted. However, it is far from reality. In fact, the estimated number of traffic accidents per year at a link can be formulated as a function of volume to capacity $(v / c)$ ratios. First, the hourly accident rate per year at a link is formulated as follows $[7,10]$ :

$$
\mu_{a}=\frac{z_{a}}{\left(365 \times v_{a} \times l_{a} / 10^{8}\right)},
$$




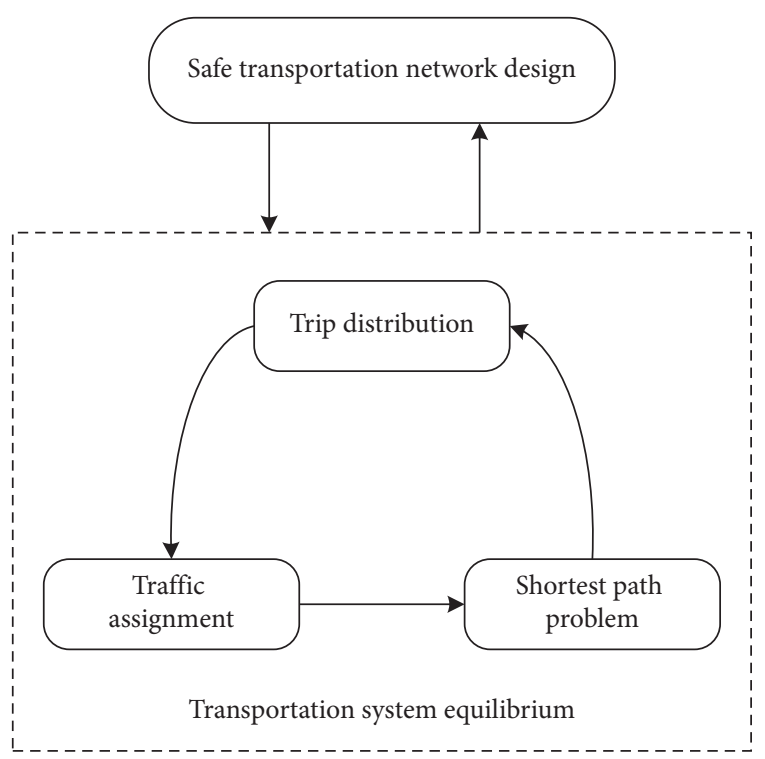

FIGURE 1: The framework for safe transportation network design.

where $\mu_{a}$ is the hourly traffic accident rate of link $a$ in terms of accidents per hundred million vehicle kilometers of travel, acc/100mvkm; $z_{a}$ is the average hourly number of accidents of link $a$ per year $(a c c)$; $v_{a}$ is the annul average hourly traffic (AAHT) volume of link $a(p c u / h) ; l_{a}$ is the length of link $a$ $(\mathrm{km})$. Note that the vehicle is regarded as passenger car unit $(p c u)$ here. After a simple transformation, the expression of $z_{a}$ can be formulated as follows:

$$
z_{a}=\frac{365 \times v_{a} \times l_{a} \times \mu_{a}}{10^{8}},
$$

which is a function of traffic accident rates $\mu_{a}$.

Furthermore, it has been proven that the traffic accident rates $\mu_{a}$ and $v / c$ ratios typically comply with a U-shape relationship $[7,10,11]$. This relationship can be described as follows:

$$
\mu_{a}\left(v_{a}, c_{a}\right)=\gamma_{1} \times\left(\frac{v_{a}}{c_{a}}\right)^{2}+\gamma_{2} \times\left(\frac{v_{a}}{c_{a}}\right)+\gamma_{3}
$$

where $c_{a}$ is link capacity on link $a(p c u / h)$ and it is the production of lane number $n_{a}$ and lane capacity $c, c_{a}=n_{a} c$; $\gamma_{1}, \gamma_{2}$, and $\gamma_{3}$ are the parameters that can be identified using the traffic survey data of the study area. For example, Zhou and Sisiopiku [10] obtained $\gamma_{1}=358.6, \gamma_{2}=-407.7$, and $\gamma_{3}=175.3$ using the traffic survey data in Michigan. This paper adopts these parameters in the numerical experiment without losing generality.

Next, by substituting (3) into (2), the average number of accidents on link $a$ per year $z_{a}$ can be determined as follows:

$$
\begin{aligned}
z_{a}\left(v_{a}, c_{a}\right) & =\frac{365 \times v_{a} \times l_{a} \times \mu_{a}\left(v_{a}, c_{a}\right)}{10^{8}} \\
& =\frac{365 \times v_{a} \times l_{a} \times\left[\gamma_{1} \times\left(v_{a} / c_{a}\right)^{2}+\gamma_{2} \times\left(v_{a} / c_{a}\right)+\gamma_{3}\right]}{10^{8}} .
\end{aligned}
$$

Furthermore, the safety performance of urban road networks is the sum of all component links. It is formulated as follows:

$$
z(\mathbf{v}, \mathbf{c})=\sum_{a \in A_{1} \cup A_{2}} z_{a}\left(v_{a}, c_{a}\right),
$$

where $z$ is the network safety performance; $A_{1}$ is the set of existing links and $A_{2}$ is the set of alternative links; $\mathbf{v}$ and $\mathbf{c}$ is the vector form of $v_{a}$ and $c_{a}$, where $a \in A_{1} \cup A_{2}$.

In order to improve the safety level of urban road network, the upper level aims to minimize the estimated total amount of accidents per year. The decision variables are the location and the number of added lanes in alternative links, including existing ones and alternative ones. To be more specific, the decision variable is the number of added lanes $n_{a}$ for each link $a \in A_{1} \cup A_{2}$. The location problem is implicitly accommodated. That is, there will be no road construction if $n_{a}=0$. The traffic flow pattern $\mathbf{v}(\mathbf{n})$ is an implicit function determined by the lower level model, where $\mathbf{v}$ is the vector form of link flows $v_{a}$ and $\mathbf{n}$ is the vector form of added number of lanes $n_{a}$. It is straightforward that the link capacity $c_{a}\left(n_{a}\right)$ is a function of $n_{a}$ and its vector form is noted as $\mathbf{c}(\mathbf{n})$. Finally, the upper level model is formulated as follows:

$$
\begin{aligned}
\operatorname{Min} z(\mathbf{v}(\mathbf{n}), \mathbf{c}(\mathbf{n})) & =\sum_{a \in A_{1} \cup A_{2}} \frac{365 \times v_{a}(\mathbf{n}) \times l_{a} \times\left[\gamma_{1}\right.}{\text { s.t. } \sum_{a \in A_{1} \cup A_{2}} g_{a}\left(n_{a}\right)} \leq B, \\
n_{a}^{\prime} & =n_{a}^{0}+n_{a}, \quad \forall a \in A_{1} \cup A_{2}, \\
n_{a}^{\prime} & \leq 3, \quad \forall a \in A_{1} \cup A_{2}, \\
c_{a}\left(n_{a}\right) & =\left(n_{a}^{0}+n_{a}\right) c, \quad \forall a \in A_{1} \cup A_{2},
\end{aligned}
$$




$$
\begin{aligned}
n_{a} & =\{0,1,2,3\}, \quad \forall a \in A_{1} \cup A_{2}, \\
y_{a} & =\left\{\begin{array}{ll}
0, & \text { if } n_{a}=0, \\
1, & \text { otherwise, }
\end{array} \quad \forall a \in A_{1} \cup A_{2},\right. \\
\sum_{a \in A_{1} \cup A_{2}} y_{a} & \leq E .
\end{aligned}
$$

The objective function in (6) is to minimize the estimated total number of traffic accidents. Equation (7) is the budget constraint that ensures the total construction cost less than the maximum allowable expenditure $B$ for network enhancement. In (7), the term $g_{a}\left(n_{a}\right)$ indicates that the link construction cost is a function with respect to the added link capacity, where $n_{a}$ is the decision variable that denotes the number of lanes to be added in single direction in link $a$. In (8), $n_{a}^{0}$ is the original number of lanes for exiting links and it should be zero for candidate links. $n_{a}^{\prime}$ is the number of improved lanes including original ones and added ones. Equation (9) ensures that the total number of improved lanes $n_{a}^{\prime}$ should less than three in single direction in urban transportation. In (10), $c$ is the single lane capacity and $c_{a}\left(n_{a}\right)$ is the link capacity. There would be no flow on a link if the link is not constructed; that is, if $n_{a}^{\prime}=0$, then $v_{a}=0$. Equation (11) indicates that the number of added lanes $n_{a}$ can only be chosen from a discrete variable $\{0,1,2,3\}$. Note that $n_{a}$ is the decision variable and this ensures its nonnegativity. In (12), $y_{a}$ is a binary variable indicating whether link $a$ is added or not. It will be zero if $n_{a}=0$ and one otherwise. Equation (13) enforces the number of links to be constructed, where $E$ is a predetermined number. It is not feasible that many links are to be constructed at the same time. It will cause serious traffic congestion and exhaust emissions.

2.2. The Lower Level Model. The lower level model is traveler behavior response to network design decisions in the upper level. It is a closed loop procedure for transportation system equilibrium. Generally speaking, there are two ways to achieve transportation system equilibrium in literature. One way is to integrate two or more steps into an equivalent single mathematical programming that ensures a wellconverged and consistent result $[12,13]$. The other way is to repeat the sequential steps until it meets the consistency requirement $[14,15]$. Although the former is widely used, it is not operation-friendly as the nonlinear programming is usually a tough problem in practice. In addition, the integrated model is less flexible as advanced models can be used in each step of the sequential models with feedback. Therefore, the latter is adopted here.

For expected travel demand in the future, trip distribution is regarded as an aggregated result of individual destination choice decisions. In literature, the multinomial logit model is the simplest and most practical random utility model used for destination choices. Although various researches have shown that the gravity analogous model and the multinomial logit model can produce equivalent results under specific suppositions, the gravity model offers a smaller behavioral base [16]. Moreover, the multinomial logit model can accommodate a number of key variables that have a significant explanatory power, including destination and individual attributes. Therefore, the multinomial logit model is adopted here to generate trip distribution matrix. Modal split is ignored as only passenger car is used for simplicity. The trip distribution matrix is assigned to road network by user equilibrium to predict link traffic flows and link travel times. Then, the shortest path travel times are fed back to the destination model to update the trip distribution matrix. The iteration process continues until the trip distribution matrix does not change to an extent. To be more specific, the feedback process at lower level is shown in Figure 2.

The notations are consistent with the previous definition. In addition, $O_{r}$ is the total travel demand in node $r . q_{r s}$ is the distributed travel demand from node $r$ to node $s . \beta_{s}$ is the alternative specific constant that is the intrinsic attractiveness of node $s . t_{r s}$ is the shortest path travel time between an origin-destination pair $r s$ and $\beta_{t}$ is the corresponding coefficient. $D_{s}$ is the attractiveness of node $s$ that is usually measured by the number of employees, and $\beta_{d}$ is the corresponding coefficient. $S r$ is the set of destinations departed from node $r . v_{a}$ is the traffic volume on link $a$ and its vector form is v. $f_{k}^{r s}$ is the traffic flow on path $k$ connecting node $r$ and node $s . \delta_{a, k}^{r s}$ is the link-path incidence relationship that is expressed as follows:

$$
\delta_{a, k}^{r s}= \begin{cases}1, & \text { if a is on path } k \text { linking } r \text { and } s \\ 0, & \text { if not. }\end{cases}
$$

The notation $t_{a}$ is a link travel time that is a function of traffic flow and capacity. In order to accommodate congestion effect, a link impedance cost function developed by the Bureau of Public Roads (BPR), a precursor of the United States Federal Highway Administration, is usually used with the following formulation:

$$
t_{a}\left(x_{a}, n_{a} c\right)=t_{a}^{0}\left[1+\alpha\left(\frac{x_{a}}{n_{a}^{0} c+n_{a} c}\right)^{\beta}\right], \quad a \in A_{1} \cup A_{2}
$$

where $t_{a}^{0}$ is free flow travel time; $\alpha$ and $\beta$ are volume/delay coefficients that can be calibrated empirically. Note that $n_{a}$ could be zero, which means there is no link added. In order to avoid the denominator being zero, a small enough positive value can be used to substitute zero. Then, the link travel 


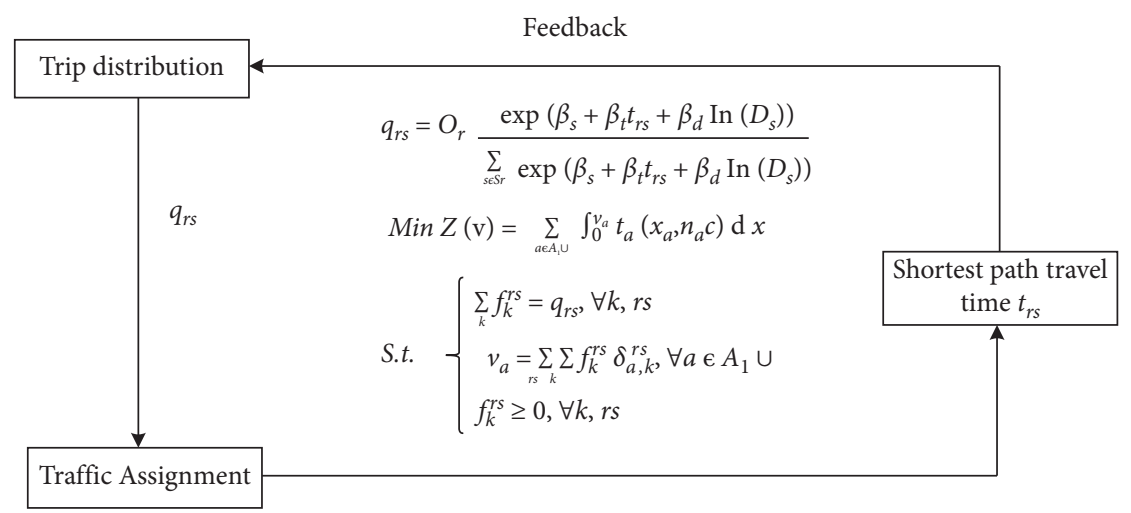

FIgURE 2: The feedback process of lower level model.

time will be big enough to ensure that there are no traffic flows and no investment cost.

\section{Solution Algorithm}

3.1. The Lower Level Algorithm. The lower level model is nested in the upper level model. In order to solve the proposed bilevel model, it is always beneficial to solve the lower level model first. Given a network enhancement pattern from the upper level, there will be a stable flow pattern generated from the lower level. Method of successive averages (MSA) is a kind of convex combinations method that can be used to achieve transportation system equilibrium in the lower level [13]. An initial trip distribution matrix can be produced by multinomial logit model with initialized path travel times. The trips are assigned road network based on user equilibrium. The link travel flows and link travel times can be produced by the conventional Frank-Wolfe algorithm [13]. According to Wardrop's first principle of route choice, also known as user equilibrium, traffic arranges itself in congested networks such that all used paths between an origin-destination pair have an equal and minimum cost. Then, the shortest path algorithm, Dijkstra algorithm, is used to update path travel times. These path times are fed back to the multinomial logit model to generate a new trip distribution matrix. However, this matrix cannot be assigned to road network directly. The convergence of direct or naive feedback is usually impossible. An averaging of successive trip distribution matrix is necessary. Although there are some successful applications of constant weights, the convergence is usually not guaranteed. Therefore, the method of successive averages with decreasing weight is used here to update trip distribution matrix, where the weight is the reciprocal of iteration number. The updated matrix is further assigned road network. The iteration process continues until the successive matrices are quasi-equal. The convergence is generally measured by the squared root of relative gap. If a predetermined tolerance is achieved, terminate the iteration. The stable state is known as transportation system equilibrium. The resultant traffic flows are then fed back to the upper level to evaluate safety performance. The flowchart of lower level algorithm is shown in Figure 3. follows:

The detailed MSA algorithm is specified in steps as
Step 1. Input a feasible network design pattern from the upper level.

Step 2. Initialize trip distribution matrix $q_{r s}^{0}$ with initial path travel time $t_{r s}^{0}$. In addition, let $n=1$ be the number of iterations.

Step 3. Traffic Assignment. Trip distribution matrix $q_{r s}^{0}$ is assigned to road network by Frank-Wolfe algorithm. The link travel flows $v_{a}$ and link travel times $t_{a}$ are generated.

Step 4. Update the shortest path travel time between an origin-destination pair $r s$, namely, $t_{r s}^{1}$, by Dijkstra algorithm.

Step 5. Trip Distribution. The multinomial logit model is used to update trip distribution matrix $q_{r s}^{1}$ :

$$
q_{r s}^{1}=O_{r} \frac{\exp \left(\beta_{s}+\beta_{t} t_{r s}^{1}+\beta_{d} \ln \left(D_{s}\right)\right)}{\sum_{s \in S r} \exp \left(\beta_{s}+\beta_{t} t_{r s}^{1}+\beta_{d} \ln \left(D_{s}\right)\right)} .
$$

Step 6. Average trip distribution matrices $q_{r s}^{1}$ and $q_{r s}^{0}$ using decreasing weight:

$$
q_{r s}^{1}=q_{r s}^{0}+\frac{1}{n}\left(q_{r s}^{1}-q_{r s}^{0}\right)
$$

Step 7. Convergence Identification. Check convergence of trip distribution matrix using squared root of relative gap:

$$
\sqrt{\sum_{r s}\left(\frac{q_{r s}^{1}-q_{r s}^{0}}{q_{r s}^{0}}\right)^{2}<\varepsilon}
$$

where $\varepsilon$ is a predetermined tolerance. If the convergence condition is satisfied, terminate the iteration and turn to Step 9; otherwise, turn to Step 8.

Step 8. Let $q_{r s}^{0}=q_{r s}^{1}$ and $n=n+1$. Then, turn to Step 3 . Step 9. Output trip distribution matrix $q_{r s}^{1}$ and link traffic flow $v_{a}$.

3.2. The Upper Level Algorithm. In the upper level, it is to determine the link improvement location and the corresponding number of added lanes in order to minimize the 


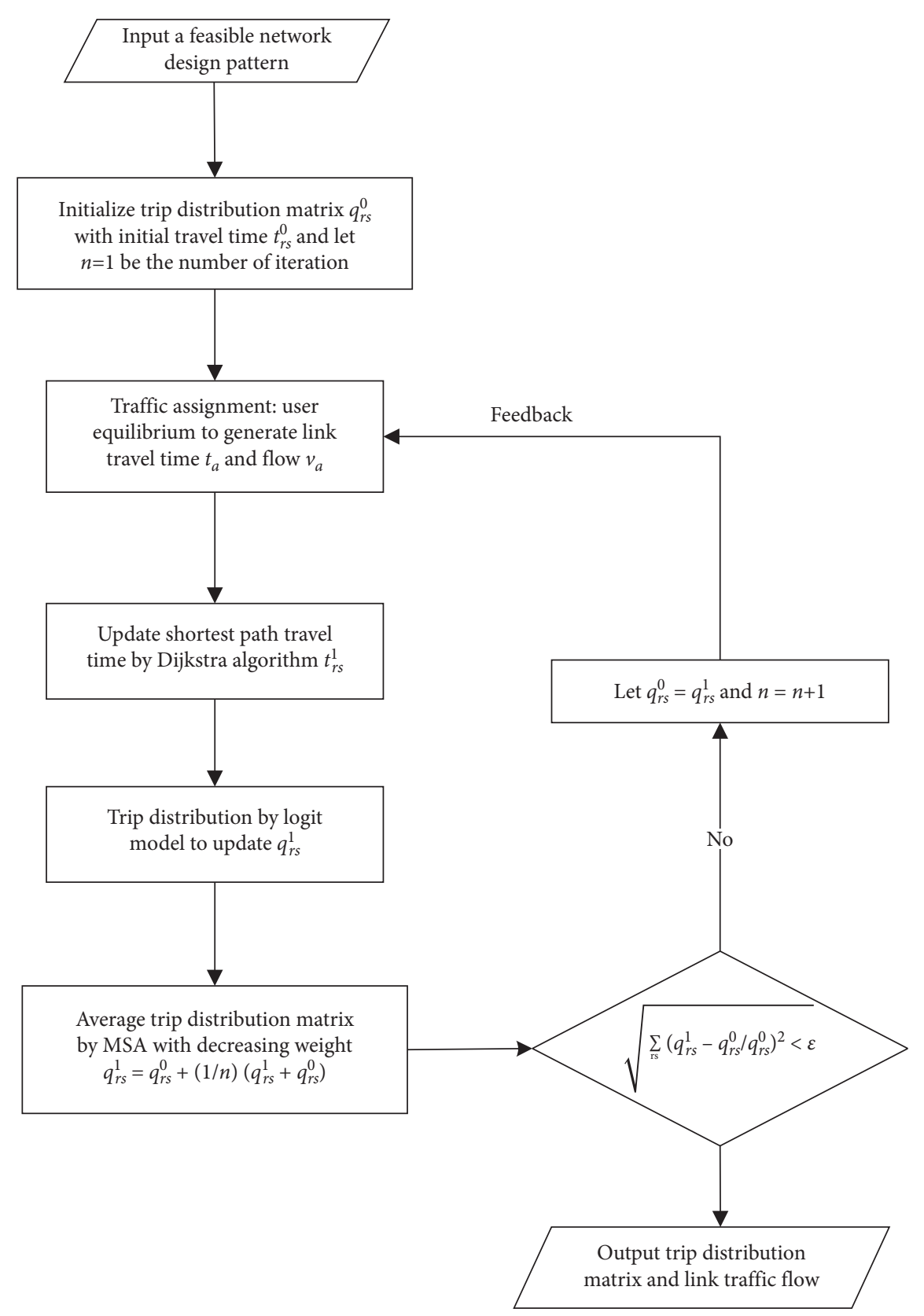

Figure 3: The flowchart of MSA algorithm.

estimated total number of traffic accidents. The bilevel model is well known to be a NP-hard problem. The global optimum is always not guaranteed by gradient-based algorithms, so that a metaheuristic algorithm is used here. Although there are kinds of metaheuristic in computer science and operations research, genetic algorithm is the most commonly used one to generate high-quality solutions. There are many successful applications for solving bilevel models in transportation research [17-19]. Therefore, genetic algorithm is adopted here. Its flowchart is shown in Figure 4.

The detailed genetic algorithm is specified in steps as follows.
Step 1. Initialization. Set the parameters used in genetic algorithm including population size $M$, the maximum number of generations Gen, crossover probability $p_{c}$, mutation probability $p_{m}$, the notation of generation gen $=1$, and the portion for elitist strategy $p_{e}$. Note that the population size depends on the nature of the problem but typically contains several hundreds of possible solutions.

Step 2. Generate a feasible initial population randomly. A gene is used to represent link location decision and another gene is used to represent lane number decision. If there is $E$ allowable links for improvement including existing ones and candidate ones, it makes a total of $2 E$ 


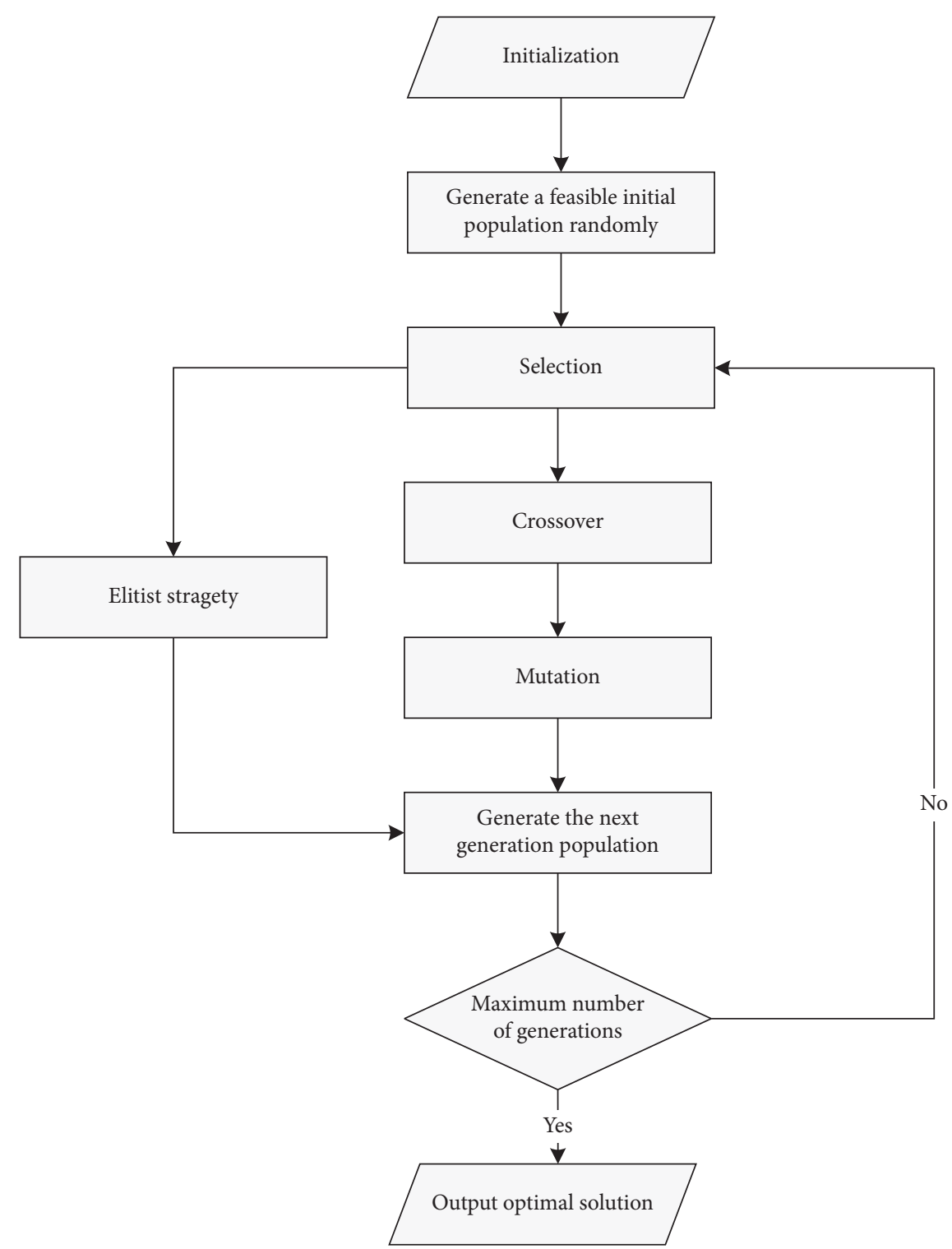

Figure 4: The flowchart of genetic algorithm.

genes in a chromosome. For example, a chromosome is depicted in Figure 5 if $E=4$.

It represents that the chosen link locations are 2, 15, 21, and 7 . The corresponding added lane number is $0,3,1$, and 2 , respectively. Generate a chromosome randomly. If it is not feasible, generate another one until it is feasible. A total number of $M$ feasible chromosomes are generated scattering the entire range of possible solutions.

Step 3. Selection Operation. For a chromosome in the upper level, that is, the network design decision, there is a corresponding transportation system equilibrium in the lower level. The lower level algorithm is used to generate link traffic flows. The objective function of the upper level is used to work as a fitness function to evaluate the performance of each chromosome in the population. Note that to minimize the total number of

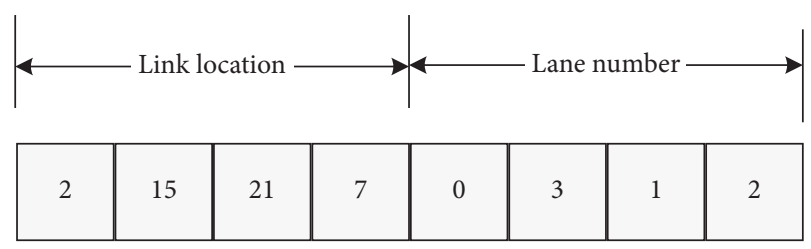

FIgURE 5: An illustration of encoding for the upper level decisions.

traffic accidents, the best $p_{e}$ is labeled for elitists and the worst $p_{e}$ is discarded.

Step 4. Crossover Operation. The remaining $\left(1-p_{e}\right) M$ chromosomes are used for crossover operation. These parent chromosomes are matched in pairs randomly. The probability to carry out crossover is $p_{c}$. If it is chosen for crossover, a random gene is identified. If new born chromosomes are not feasible according to 
constraints in the upper letter, try another gene location until they are feasible. The new solutions typically share many of the characteristics of their parents.

Step 5. Mutation Operation. The probability to carry out mutation is $p_{m}$. A random gene is identified for mutation within the domain of definition. If the new chromosome is not feasible, try another gene location until it is feasible.

Step 6. Generate the next generation population. After genetic operators, there are still $\left(1-p_{e}\right) M$ feasible chromosomes. The labeled $p_{e} M$ elitists are added to ensure the population size $M$. This allows the best chromosomes from the current generation to carry over the next unaltered. It guarantees that the solution quality will not decrease from one generation to the next. Let the notation of generation be gen $=$ gen +1 .

Step 7. Termination Judgment. If the maximum number of generations is achieved, that is, gen $\geq$ Gen, terminate the iteration process and output the optimal network design. Otherwise, turn back to Step 3.

\section{Experimental Study}

The Nguyen-Dupuis road network as shown in Figure 6 is commonly used in transportation research to demonstrate various methods and algorithms. The link characteristics including free flow travel time, link capacity, and link length are shown in Table 1.

There are two origin nodes 1 and 4 and two destination nodes 2 and 3 in the Nguyen-Dupuis network. The predicted travel demands at origin nodes 1 and 4 are 2,000 pcu/h and $2,000 \mathrm{pcu} / \mathrm{h}$, respectively. That is, $O_{1}=2,000 \mathrm{pcu} / \mathrm{h}$ and $\mathrm{O}_{4}$ $=2,000 \mathrm{pcu} / \mathrm{h}$. All of existing links, labeled from 1 to 19 , and candidate links, labeled from 20 to 24 , are ready for capacity enhancements. The problem is to identify the optimal link enhancement pattern in terms of network safety performance in order to make best use of the investment budget.

The parameters used in the lower level algorithm are summarized as follows. The multinomial logit model for destination choices is simplified as follows:

$$
q_{r s}^{1}=O_{r} \frac{\exp \left(\beta_{s}+\beta_{t} t_{r s}^{1}\right)}{\sum_{s \in S r} \exp \left(\beta_{s}+\beta_{t} t_{r s}^{1}\right)},
$$

where $\beta_{s}$ is the traveler intrinsic preference on destination $s$ and $\beta_{t}$ is the coefficient of path travel time between O-D pair rs. The values of $\beta_{s}$ and $\beta_{t}$ can be calibrated empirically. Here, we set $\beta_{2}=0, \beta_{3}=1$, and $\beta_{t}=-0.1$. That is, the traveler preference on destination node 2 is 0 and on destination node 3 is 1 , which means that the travelers traditionally prefer destination 3 . The coefficient of travel time is -0.1 , which means that the travel time is a negative utility. In addition, the parameters in (14) for traffic assignment are set as $\alpha=0.15$ and $\beta=4$, conventionally. The convergence criteria for MSA are set as $\varepsilon=0.01$. A stable transportation system can be achieved for an upper level decision.
The parameter tuning for metaheuristic algorithms is usually a tricky problem. The parameters used in the genetic algorithm are listed as follows. The portion for elitists is $p_{e}=0.1$. The crossover probability is $p_{c}=0.1$, and the mutation probability is $p_{m}=0.5$. Although these parameters are conventionally used in genetic algorithm, it is worth tuning parameters to find reasonable settings for the problem. The link construction cost is assumed to be a linear function of link length and unit lane cost. It is formulated as follows:

$$
g_{a}\left(n_{a}\right)=b_{a} \times n_{a} \times l_{a}, \quad a \in A_{1} \cup A_{2},
$$

where $l_{a}$ is the link length in kilometer and $b_{a}$ is the unit lane cost that is assumed to be 0.5 million dollars. The investment budget is set as $B=30$ million dollars. The maximum number of construction links is $E=4$. The capacity for a single lane is $c=800 \mathrm{pcu} / \mathrm{h}$. The population size $M$ and generation size Gen are the major factors affecting computation time. Although a better solution can be reached with bigger population and generation size, it could be timeconsuming. For this reason, it is usually recommended to run GA multiple times to trade off the quality of solutions and parameters. Therefore, varying population and generation sizes are tested to identify the best parameters as shown in Table 2.

The calculation is programmed using a popular open source language $R$ 3.6.1 in a personal computer with Intel Core i7-4790 CPU @ 3.60 GHz. It is no doubt that the computation time increases with population size and generation size. Although the computation time varies from 1.68 hours to 10.49 hours, the quality of solutions does not improve a lot for this small-scale problem. The exact result could be around 32, and they all close to exact result. For the sake of computation time, the population size could be set as $M=100$, and the generation size could be set as Gen $=10$. The optimal link enhancement pattern and transportation system performances at this time are shown in Table 3.

The link label for lane addition is 4 and 16 among existing links. They are expected to increase 1 and 2 more lanes, respectively. The candidate links 20 and 22 are expected to be built with 2 and 3 lanes, respectively. At this time, the investment cost is 29 million dollars, which does not exceed the budget constraint. The number of traffic accidents is 32.76 after investment, while the estimated number of traffic accidents is 76.50 before investment. Therefore, the network safety performance is significantly improved after investment.

It is always beneficial to do a sensitivity analysis. The sensitivity of objective value with respect to the major constraint factor, investment budget $\mathrm{B}$, is investigated. The budget is increased from 15 million dollars to 50 million dollars by Step 5 million dollars. The corresponding accident numbers are shown in Figure 7 . It shows that the accident numbers decrease with investment. However, it decreases quickly at the beginning and then becomes slow especially after 30 million dollars. It is no wonder as four links are allowed to expand. The system performance cannot increase continuously with a small part improved. It is wasteful to 


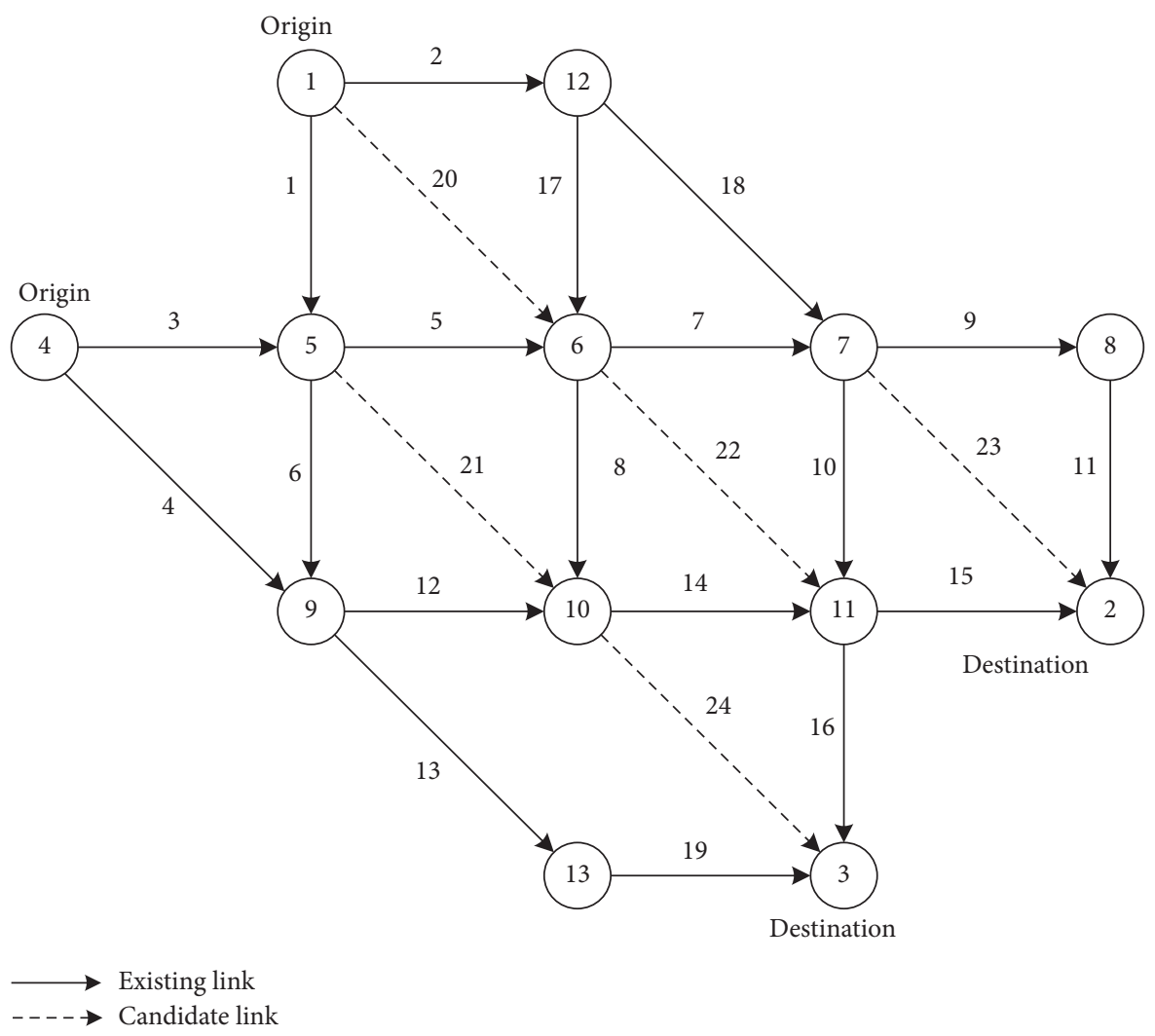

Figure 6: The Nguyen-Dupuis road network.

TABLe 1: Link characteristics of the Nguyen-Dupuis road network.

\begin{tabular}{lccc}
\hline Link $a$ & Free flow time $(\mathrm{min})$ & Link capacity $(p c u / h)$ & Link length $(\mathrm{km})$ \\
\hline 1 & 7.0 & 800 & 4.00 \\
2 & 9.0 & 800 & 6.00 \\
3 & 9.0 & 800 & 5.00 \\
4 & 12.0 & 800 & 8.00 \\
5 & 3.0 & 800 & 2.00 \\
6 & 9.0 & 800 & 5.00 \\
7 & 5.0 & 800 & 3.00 \\
8 & 13.0 & 800 & 8.00 \\
9 & 5.0 & 800 & 3.00 \\
10 & 9.0 & 800 & 6.00 \\
11 & 9.0 & 800 & 5.00 \\
12 & 10.0 & 800 & 6.00 \\
13 & 9.0 & 800 & 5.00 \\
14 & 6.0 & 800 & 4.00 \\
15 & 9.0 & 800 & 6.00 \\
16 & 8.0 & 800 & 5.00 \\
17 & 7.0 & 800 & 4.00 \\
18 & 14.0 & 800 & 6.00 \\
19 & 11.0 & 800 & 7.00 \\
20 & 12.0 & 0 & 8.00 \\
21 & 12.0 & 0 & 8.00 \\
22 & 12.0 & 0 & 8.00 \\
23 & 12.0 & 0 & 8.00 \\
24 & 12.0 & 0 & 8.00 \\
\hline
\end{tabular}


TABLE 2: Multiple GA tests with varying population and generation size.

\begin{tabular}{lcccc}
\hline Population size & Generation size & Investment (million dollars) & Computation time (hours) & Accident number \\
\hline 100 & 10 & 29 & 1.68 & 32.76 \\
100 & 20 & 26 & 3.96 & 33.14 \\
200 & 10 & 28 & 2.93 & 34.75 \\
200 & 20 & 30 & 5.39 & 3.49 \\
300 & 10 & 29 & 10.49 & 33.49 \\
300 & 20 & 30 & & 32.13 \\
\hline
\end{tabular}

TABle 3: The optimal network design and link performances.

\begin{tabular}{|c|c|c|c|c|}
\hline Link & Lane addition (no.) & Road capacity $(p c u / h)$ & Traffic volume $(p c u / h)$ & Travel time $(\mathrm{min})$ \\
\hline 1 & 0 & 800 & 543 & 7.22 \\
\hline 2 & 0 & 800 & 0 & - \\
\hline 3 & 0 & 800 & 737 & 9.97 \\
\hline 4 & 1 & 1.600 & 1,263 & 12.70 \\
\hline 5 & 0 & 800 & 1,280 & 5.95 \\
\hline 6 & 0 & 800 & 0 & - \\
\hline 7 & 0 & 800 & 776 & 5.66 \\
\hline 8 & 0 & 800 & 0 & - \\
\hline 9 & 0 & 800 & 776 & 5.66 \\
\hline 10 & 0 & 800 & 0 & - \\
\hline 11 & 0 & 800 & 776 & 10.20 \\
\hline 12 & 0 & 800 & 364 & 10.06 \\
\hline 13 & 0 & 800 & 899 & 11.15 \\
\hline 14 & 0 & 800 & 364 & 6.04 \\
\hline 15 & 0 & 800 & 291 & 9.02 \\
\hline 16 & 2 & 2,400 & 2,033 & 8.62 \\
\hline 17 & 0 & 800 & 0 & - \\
\hline 18 & 0 & 800 & 0 & - \\
\hline 19 & 0 & 800 & 899 & 13.63 \\
\hline 20 & 2 & 1,600 & 1,457 & 13.24 \\
\hline 21 & 0 & 0 & 0 & - \\
\hline 22 & 3 & 2,400 & 1,961 & 12.80 \\
\hline 23 & 0 & 0 & 0 & - \\
\hline 24 & 0 & 0 & 0 & - \\
\hline
\end{tabular}

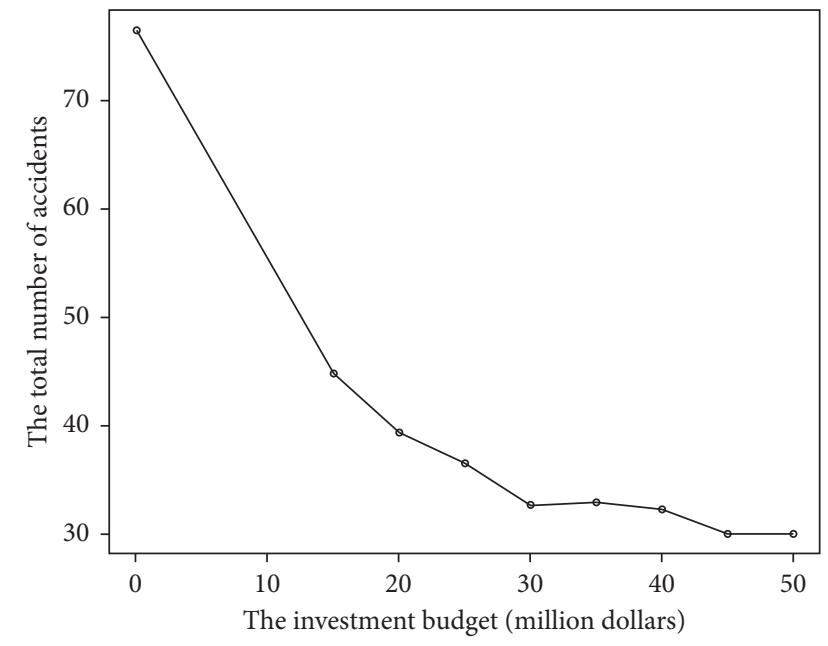

FIGURE 7: The sensitivity of objective value with respect to varying budget. invest as much as we can. The policy makers have to determine the best investment budget to make good use of the public funds.

\section{Conclusions}

This paper proposed a method for active safety planning at the transportation network design stage. It is a bilevel programming model system. Urban planners in the upper level optimize the network safety performance in terms of estimated number of traffic accidents. The travelers in the lower level make behavior responses for the upper decision. It is a feedback procedure between destination choice and traffic assignment in order to achieve transportation system equilibrium. Note that the urban planners have to take the behavior responses of travelers into consideration to adjust their decisions for system optimization.

The bilevel model is a well-known challenging problem. A genetic algorithm with elite strategy is proposed where 
MSA is embedded for the lower level model. The algorithm aims to provide a feasible solution at costs that can be accepted. It is worth noting that the metaheuristics cannot guarantee the optimal solution. However, these approaches can solve large-scale nonlinear problems within the appropriate time. Therefore, developing this kind of solution algorithms is worthwhile.

The experimental study shows that the proposed methods are effective for safe transportation network design although system total travel time is sacrificed to a certain extent. However, a real-world case study is expected in the future, which could be more convincing.

\section{Data Availability}

The data used to support the experimental study are included within the article.

\section{Conflicts of Interest}

The author declares no conflicts of interest.

\section{Acknowledgments}

This work was supported by the National Social Science Fund of China (no. 21BGL233).

\section{References}

[1] H. Huang, P. Xu, M. Ma, and M. Abdel-Aty, "Recent theoretical researches on transportation safety planning," China Journal of Highway and Transport, vol. 27, pp. 90-97, 2014.

[2] Safetea-Lu, "Safe, accountable, flexible, efficient transportation equity Act: a legacy for users," U.S. Federal Highway Administration, Washington, DC, USA, SAFETEA-LU, 2005.

[3] Y. Yang, H. Lu, Y. Yin, and H. Yang, "Optimization of variable speed limits for efficient, safe, and sustainable mobility," Transportation Research Record: Journal of the Transportation Research Board, vol. 2333, no. 1, pp. 37-45, 2013.

[4] X. Xu, A. Chen, and L. Cheng, "Stochastic network design problem with fuzzy goals," Transportation Research Record: Journal of the Transportation Research Board, vol. 2399, no. 1, pp. 23-33, 2013.

[5] E. Rashidi, M. Parsafard, H. Medal, and X. Li, "Optimal traffic calming: a mixed-integer bi-level programming model for locating sidewalks and crosswalks in a multimodal transportation network to maximize pedestrians' safety and network usability," Transportation Research Part E: Logistics and Transportation Review, vol. 91, pp. 33-50, 2016.

[6] I. Haas and S. Bekhor, "Network design problem considering system time minimization and road safety maximization: formulation and solution approaches," Transportmetrica: Transportation Science, vol. 13, no. 9, pp. 829-851, 2017.

[7] S. Zhong, X. Xiao, M. Bushell, and H. Sun, "Optimal road congestion pricing for both traffic efficiency and safety under demand uncertainty," Journal of Transportation Engineering, vol. 143, 2017.

[8] B. Possel, L. J. J. Wismans, E. C. Van Berkum, and M. C. J. Bliemer, "The multi-objective network design problem using minimizing externalities as objectives: comparison of a genetic algorithm and simulated annealing framework," Transportation, vol. 45, no. 2, pp. 545-572, 2018.
[9] H.-Z. Lin and J. Wei, "Optimal transport network design for both traffic safety and risk equity considerations," Journal of Cleaner Production, vol. 218, pp. 738-745, 2019.

[10] M. Zhou and V. P. Sisiopiku, "Relationship between volumeto-capacity ratios and accident rates," Transportation Research Record: Journal of the Transportation Research Board, vol. 1581, no. 1, pp. 47-52, 1997.

[11] J.-L. Martin, "Relationship between crash rate and hourly traffic flow on interurban motorways," Accident Analysis \& Prevention, vol. 34, no. 5, pp. 619-629, 2002.

[12] N. Oppenheim, Urban Travel Demand Modeling: From Individual Choices to General Equilibrium, John Wiley \& Sons, Hoboken, NJ, USA, 1995.

[13] Y. Sheffi, Urban Transportation Networks: Equilibrium Analysis with Mathematical Programming Methods, PrenticeHall, Englewood Cliffs, NJ, USA, 1985.

[14] D. Boyce, C. R. O'Neill, and W. Scherr, "Solving the sequential travel forecasting procedure with feedback," Transportation Research Record: Journal of the Transportation Research Board, vol. 2077, no. 1, pp. 129-135, 2008.

[15] D. E. Boyce, Y.-F. Zhang, and M. R. Lupa, "Introducing "feedback" into four-step travel forecasting procedure versus equilibrium solution of combined model," Transportation Research Record, vol. 1443, pp. 65-74, 1994.

[16] R. Cordera, A. Ibeas, L. dell'Olio, and B. Alonso, Land Use Transport Interaciton Models, CRC Press, Boca Raton, FL, USA, 2018.

[17] Y.-C. Chiou, L. W. Lan, and K.-L. Chang, "Sustainable consumption, production and infrastructure construction for operating and planning intercity passenger transport systems," Journal of Cleaner Production, vol. 40, pp. 13-21, 2013.

[18] Y. Yin, "Genetic-algorithms-based approach for bilevel programming models," Journal of Transportation Engineering, vol. 126, no. 2, pp. 115-120, 2000.

[19] X. Zhang, S. T. Waller, D. Rey, and M. Duell, "Integrating uncertainty considerations into multi-objective transportation network design projects accounting for environment disruption," Transportation Letters, vol. 11, no. 7, pp. 351-361, 2019. 